\title{
Analysis of Data Log
}

National Cancer Institute

\section{Source}

National Cancer Institute. Analysis of Data Log. NCI Thesaurus. Code C102867.

A record of data captured about device logging functions, error logs, keystroke logs and device alarm history. 